\title{
Refactoring Business Process Models A Systematic Review
}

\author{
María Fernández-Ropero, Ricardo Pérez-Castillo and Mario Piattini \\ Instituto de Tecnologías y Sistemas de Información (ITSI), University of Castilla-La Mancha, \\ Paseo de la Universidad 4, 13071, Ciudad Real, Spain \\ \{MariaS.Fernandez, Ricardo.PdelCastillo,Mario.Piattini\}@uclm.es
}

Keywords: Refactoring, Business Process, Systematic Literature Review.

\begin{abstract}
Business processes are nowadays recognized as one of the intangible business assets that provide more competitive advantage to organizations. Organizations must therefore be able to manage their business process models and deal with their quality problems, i.e. lack of understandability, maintainability or reusability among others. Such quality problems are exacerbated in business processes models that were mined by reverse engineering from enterprise information systems, since business process are more likely to undergo inconsistencies, redundancies, etc. Refactoring has proved to be a suitable solution to cope with these quality problems. Refactoring changes the internal structure of a business process model while preserves its external behaviour. This paper presents an in-depth systematic review for collecting, categorizing and analyzing all the refactoring methods and techniques applied to business process models. The systematic review is conducted following the formal methodology proposed by Kitchenhan. The review reports 206 related studies, from which 16 were considered as primary studies. The most valuable conclusion is that none of these studies proposes refactoring techniques for business process models previously obtained by reverse engineering, which is considered as a greenfield research area.
\end{abstract}

\section{INTRODUCTION}

Business processes depict sequences of coordinated business activities as well as the involved roles and resources that organizations must carry out to achieve common goals (Weske, 2007). Business processes are today recognized as an intangible business asset that provides competitive advantages for organizations (Jeston et al., 2008). In addition, most business processes are automated by enterprise information systems.

To take an effective advantage of business process management, business processes need to be represented in models following standard notations such as BPMN (Business Process Modeling and Notation) (OMG, 2006). Organizations sometimes do not explicitly have their business processes models because they have never modeled their business processes before. Even when the organization has business process models, such models can be outdated or misaligned regarding the actual processes supported by enterprise information systems. In these cases, reverse engineering techniques can be used to obtain business process models from existing information systems (PérezCastillo et al., 2011a, Pérez-Castillo et al., 2011b). This way, the retrieved models are prone to have a lower quality degree since every reverse engineering technique is characterized by a semantic loss when the abstraction level is progressively increased (in this case from existing information systems to business processes).

In fact, bussiness process models can sometimes present quality faults such as redundancies, ambiguities, inconsistencies, lack of completeness, as well as non-adherence to conventions or standards, among others (Mens et al., 2007). Business process models particularly obtained by reverse engineering dramatically face these problems. For this reason its is necessary to carry out a refactoring process, which can solve the mentioned quality problems. Refactoring modifies the internal structure of business process models without changing or altering the external semantics. Refactoring techniques therefore make it possible to improve the quality of business processes, so that they become more understandable, maintenible and reusable (Dijkman et al., 2011). 
The concept of refactoring was proposed by Opdyke in 1992 as a methodology for restructuring programs (Opdyke, 1992). In the last decades refactoring has emerged as a technique to improve the maintainability of software systems. Traditional refactoring primarily focuses on the level of source code for the software life cycle, but it can also be applied to the scope of the models (Mens and Tourwé, 2004). For example, refactoring has been applied to UML (Unified Modeling Language) models and also to business process modeling.

Refactoring is also frequently termed as restructuring since it is applied as the second stage of software modernization process. Software modernization advocates carrying out traditional software reengineering following the model-driven developments principles (i.e., considering all the involved artifacts as models at different abstraction levels). Software modernization consists of three stages: (i) reverse engineering, which represents the system at a higher level of abstraction; (ii) restructuring or refactoring, which represents the system at the same level of abstraction, improving one or more properties of the system, preserving its external behavior; and (iii) forward engineering, which generates the implementation of the system at a lower level of abstraction integrating the new features included in the previous stage.

Business process models refactoring, within a software modernization process, first needs to detect refactoring opportunities and then it applies refactoring operators. Currently, there are several techniques and approaches to refactor business process models. However, there is not a systematic review of all available techniques in the literature in order to understand its evolution and motivation in each case. For this reason this paper presents a systematic literature review to know the refactoring techniques and detect those techniques that can be particularly applied to business process models retrieved by reverse engineering. The review provides a summary of the state-of-the-art and identifies possible areas of research that have not been addressed yet.

The systematic literature review is planed and conducted following the process proposed by (Kitchenham and Charters, 2007) which is organized in: (1) planning, which defines the research questions and establishes the search protocol; (2) execution, in which the search of research studies is systematically carried out according to the protocol defined in the previous phase; and (3) analysis of results, in which some of the retrieved studies are considered as primary and are analyzed to draw conclusions.

After analyzing the studies retrieved during the search, the following main conclusions were obtained: (1) studies about business process refactoring hardly ever provide an empirical validation of their proposals, which reveals that this research field is not mature enough; (2) there is an increasing interest in the area in recent years; and (3) none of the studies proposes to refactor business process models previously obtained through reverse engineering from existing information systems. As a result, there is a potential research field that has not been addressed by now.

The remaining of the paper is organized as follows: Section 2 describes the first phase of the systematic review through the formulation of research questions. Section 3 corresponds to the second phase of the systematic review, the conduction. Section 4 describes the analysis of the results, concluding the process of Kitchenham. Finally, Section 5 shows the obtained conclusions after the execution of a systematic literature review.

\section{PLANNING THE REVIEW}

This section presents the planning of the review. It shows the research questions formulated and the development of the protocol to guide the review.

\section{$2.1 \quad$ Research Questions}

This section provides the research questions formulated in the review, which must be answered after analysing the obtained results. Table 1 shows the research questions RQ1 and the additional question AQ1.

\subsection{Development of the Protocol}

This section specifies the method used to carry out the systematic review. The review protocol provides guidelines to find primary studies that give an answer to the research questions. Thus, it is necessary to have some selection criteria of these primary studies for their inclusion in or exclusion from the systematic review. Then, a categorization of each primary study is performed through data extraction.

\subsubsection{Formulation of the Search Strings}

In order to formulate the search string it is necessary to know what to search and where to search. 
The answer to the first question is obtained from search terms that are derived from the research questions ('Refactoring' and 'Business Process Model'), together with the related terms that are included in the search string by using the logical OR. Some of these terms are subsets of other terms. For this reason, it has been decided to select the most general terms in order to avoid redundancies. The search string is shown in Table 2.

As regards to where to search it is necessary to establish a series of digital libraries where the searches will be performed. The search of the string mentioned above is performed in the following digital libraries: (DL1) ACM Digital Library, (DL2) Springer Link, (DL3) IEEE Xplore Digital Library and (DL4) Scopus. Each of these digital libraries provides a different search, so it is necessary to make small changes in the search string in order to adapt it to each of these mechanisms.

\subsubsection{Selection Criteria}

This section defines the inclusion and exclusion criteria that must pass each of the studies retrieved by the search string in order to be considered as primary studies.

Inclusion criteria are those that determine if a study is considered for review or not (see Table 3), while exclusion criteria are applied after them to exclude non-relevant studies (see Table 4).

\subsubsection{Study Selection Procedure}

The procedure to retrieve primary studies for the systematic review is shown in Table 5 .

\section{CONDUCTING THE REVIEW}

After planning the systematic literature review by the search protocol the primary studies are obtained by its execution. The steps for conducting the review are:

1. Adapt the search string for each digital library, since each one has different search engines.

2. Carry out a search in each of the digital libraries.

3. Apply de procedure of study selection to obtain the primary studies.

4. Use the data extraction mechanism for managing the relevant information from the studies.
Table 1: Research Questions.

\begin{tabular}{|c|l|}
\hline Id. & \multicolumn{1}{|c|}{ Research Questions } \\
\hline RQ1 & $\begin{array}{l}\text { What techniques or procedures exist to carry } \\
\text { out refactoring business process models? }\end{array}$ \\
\hline AQ1 & $\begin{array}{l}\text { Are there any techniques or procedures to } \\
\text { refactor business process models obtained } \\
\text { through reverse engineering? }\end{array}$ \\
\hline
\end{tabular}

Table 2: Research String.

\begin{tabular}{|c|l|}
\hline Id. & \multicolumn{1}{|c|}{ Research String } \\
\hline RS1 & $\begin{array}{l}\text { (Refactoring OR Restructuring OR Refactored } \\
\text { OR Refactor) AND (Process Model OR BPMN } \\
\text { OR Workflows OR Business Process) }\end{array}$ \\
\hline
\end{tabular}

Table 3: Inclusion Criteria.

\begin{tabular}{|c|l|}
\hline Id. & \multicolumn{1}{|c|}{ Inclusion Criteria } \\
\hline IC1 & $\begin{array}{l}\text { The study answers directly to the research } \\
\text { question }\end{array}$ \\
\hline IC2 & $\begin{array}{l}\text { The study is focused on the refactoring of } \\
\text { business process models }\end{array}$ \\
\hline IC3 & $\begin{array}{l}\text { The study has been published between January } \\
\text { 2006 and December 2011 }\end{array}$ \\
\hline IC4 & The study provides empirical validation \\
\hline
\end{tabular}

Table 4: Exclusion Criteria.

\begin{tabular}{|c|l|}
\hline Id. & \multicolumn{1}{|c|}{ Exclusion Criteria } \\
\hline EC1 & The study has a business focus \\
\hline EC2 & $\begin{array}{l}\text { The study has an approach away from software } \\
\text { engineering }\end{array}$ \\
\hline EC3 & The study is duplicated \\
\hline EC4 & $\begin{array}{l}\text { The study is written in a language different to } \\
\text { English }\end{array}$ \\
\hline EC5 & $\begin{array}{l}\text { The study shows only personal opinions or } \\
\text { anecdotes without scientific basis }\end{array}$ \\
\hline
\end{tabular}

Table 5: Procedure for studies selection.

1. Read Title and abstract of study $\mathrm{e}_{\mathrm{i}}$

2. Apply Inclusion Criteria (IC)

2.1. If $\forall \mathrm{c} \in \mathrm{IC}, \mathrm{e}_{\mathrm{i}}$ SATISFIES $\mathrm{c}$ go to step 3

2.2. If $\exists \mathrm{c} \in \mathrm{IC}, \mathrm{e}_{\mathrm{i}} \neg$ SATISFIES $\mathrm{c}$ go to step 8

2.3. If not enough information to determine it, go to step 3

3. Apply Exclusion Criteria (EC)

3.1. If $\forall \mathrm{c} \in \mathrm{EC}, \mathrm{e}_{\mathrm{i}} \neg$ SATISFIES $\mathrm{c}$ go to step 4

3.2. If $\exists \mathrm{c} \in \mathrm{EC}, \mathrm{e}_{\mathrm{i}} \mathrm{SATISFIES} \mathrm{c}$ go to step 8

3.3. If not enough information to determine it, go to step 4

4. Read full text

5. Apply Inclusion Criteria again (IC)

5.1. If $\forall \mathrm{c} \in \mathrm{CI}, \mathrm{e}_{\mathrm{i}}$ SATISFIES $\mathrm{c}$ go to step 6

5.2. If $\exists \mathrm{c} \in \mathrm{CI}, \mathrm{e}_{\mathrm{i}} \neg$ SATISFIES $\mathrm{c}$ go to step 8

6. Apply Exclusion Criteria again (EC)

6.1. If $\exists \mathrm{c} \in \mathrm{CE}, \mathrm{e}_{\mathrm{i}}$ SATISFIES $\mathrm{c}$ go to step 8

6.2. If $\forall \mathrm{c} \in \mathrm{CE}, \mathrm{e}_{\mathrm{i}} \neg \mathrm{SATISFIES} \mathrm{c}$ go to step 7

7. Accept study

8. Refuse study 
When applying the search protocol it is necessary to extract the relevant information from each retrieved study. To manage the information from each of the studies it has been prepared a form of relevant information that collects the relevant information for each retrieved study as an identifier of the study, the digital library where the study was recovered, the title of the study, the list of authors of the study and the year of publication of the study. Also, the form includes information relating to the satisfaction of the inclusion and exclusion criteria.

For each primary study, additional relevant information is also stored for later analysis, i.e. publication type (journal, conference or book), used notation (BPMN, Petri Nets, PTS Tree or others) and kind of empirical validation performed in the study (experiment, study case, poll, example or no validation). Moreover, the AQ1 (see Table 1) is formulated to each of the primary studies. This additional relevant information is collected to assess the quality of each study.

All of the primary studies are indexed in the database that provides the tool EndNote (Reuters, 2011). Additionally, all collected information during the execution of the systematic review is available online in (Pérez-Castillo and Fernández-Ropero, 2012).

\section{ANALYSIS OF RESULTS}

The obtained results are analyzed to be interpreted and to draw conclusions. The systematic review was carried out with two different analyses (see the following sections): (1) a descriptive statistical analysis and (2) a state-of-the-art assessment.

\subsection{Statistics Analysis}

After the execution of the search with the search string 206 studies were retrieved and after full execution of the review 16 studies were considered as primary studies. Table 6 provides some descriptive statistics.

Regarding the inclusion/exclusion criteria, only $7.77 \%$ of the studies met the inclusion and exclusion criteria proposed at the beginning. In the case of the $A C M$ library only $28.57 \%$ of the studies were accepted as primary studies, with 4 studies published in journals, 9 published in conferences and 1 published in a book. In the case of Springer library only $4.17 \%$ of studies were accepted, with 1 published in a book. This decrease is due to the fact that most studies are duplicated in next libraries because those studies met the exclusion criterion
EC3. The same happened with IEEE Xplore library; for this reason none of studies were considered as primary studies from this library. Finally, from Scopus library only $0.96 \%$ of the retrieved studies were considered as primary studies, with 1 published in a journal, because it was the last library to search.

Concerning the kind of the publications, Table 6 shows that the majority of the studies $(56 \%)$ have been published in conferences while $31 \%$ and $13 \%$ of the studies were respectively published in journals or books. This indicates that the subject under study does not have a great maturity degree.

Considering the year of publication, Figure 1 shows that there is a large increase of publications in the last years. In fact, in 2010 there was an increase in publication, but most of them are not relevant for this review since they did not meet the inclusion criteria (as answering the research questions) or since those studies did not provide empirical validation. Regarding primary studies, a growth is observed along the years with various studies per year from 2006.

Regarding the notation used to represent business process in the primary studies, Petri Nets predominates with $50 \%$ over all the primary studies (see Figure 2 (a)). The next most predominant notation is BPMN, which is used in $19 \%$ of the primary studies. Petri Nets might be more commonly used than BPMN given that Petri Nets is a well-proven notation and it has been used since the 60 's while the BPMN notation is relatively new and it is not widespread.

With respect to the type of the empirical validation conducted in the primary studies, it shows that most studies $(63 \%)$ provide only examples (see Figure 2 (b)). The reason for this could be a certain lack of maturity in the field and only proposals have been made.

Table 6: Summary of results.

\begin{tabular}{|c|c|c|c|c|c|c|}
\hline \multirow[b]{2}{*}{$\begin{array}{l}\text { Digital } \\
\text { Library }\end{array}$} & \multirow[b]{2}{*}{$\begin{array}{c}\text { Retrieved } \\
\text { studies }\end{array}$} & \multicolumn{4}{|c|}{ Primary studies } & \multirow[b]{2}{*}{$\begin{array}{l}\text { Primary/ } \\
\text { Retrieved } \\
\text { studies }\end{array}$} \\
\hline & & $\stackrel{\widetilde{\Xi}}{\stackrel{\Xi}{\Xi}}$ & 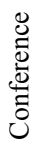 & प̆ & 岕 & \\
\hline$A C M$ & 49 & 4 & 9 & 1 & 14 & $28.57 \%$ \\
\hline Springer & 24 & 0 & 0 & 1 & 1 & $4.17 \%$ \\
\hline IEEE & 29 & 0 & 0 & 0 & 0 & $0.00 \%$ \\
\hline Scopus & 104 & 1 & 0 & 0 & 1 & $0.96 \%$ \\
\hline TOTAL & 206 & 5 & 9 & 2 & 16 & $7.77 \%$ \\
\hline
\end{tabular}

Additionally, the analysis of the primary studies revealed that no primary study considers business process models obtained through reverse engineering. 


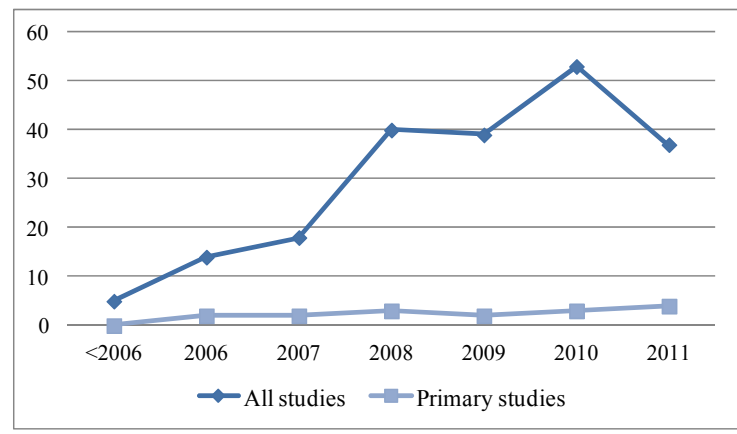

Figure 1: Distribution of year of publication.

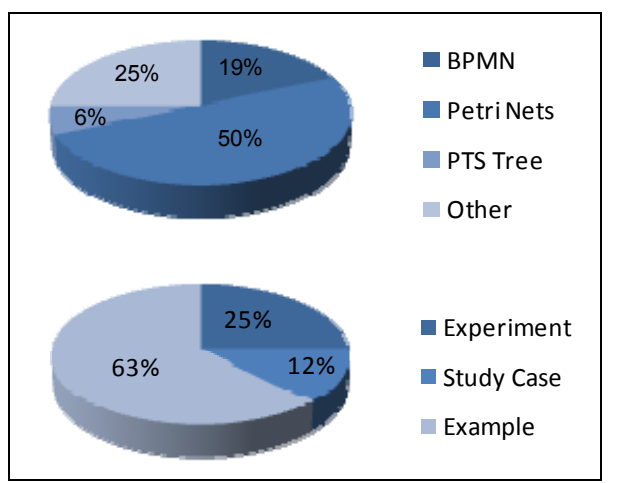

Figure 2: Distribution of primary studies according (a) notation type and (b) empirical validation type.

Finally, the location of the authors involved in the studies was also analyzed, since it may be important to understand the usefulness of the findings. At least ten countries were found, the most common of which being Germany (5 authors) as well as China and Switzerland (3 authors). Other countries as Austria, Netherlands, United Kingdom and United States have 2 authors, and Australia, Estonia and Japan have one author.

\subsection{State-of-the-Art Analysis}

After analyzing the whole set of primary studies, a set of common topics was collected. This analysis establishes a relationship between the most common, valuable topics and the primary studies addressing such topics.

Table 7 shows the reference of each primary study, that can be consulted in (Pérez-Castillo and Fernández-Ropero, 2012), and also shows the digital library where the study was retrieved and a list with all the topics indicating which topic appears or is addressed for each primary study.

There are 14 topics related to the studies. Topics are related to whether the study shows some scenarios (also called smells or refactoring opportunities) where refactoring would be necessary and, besides, if the algorithm used to make the detection is shown. Moreover, topics related to whether the study shows refactoring techniques and its algorithm. Other topic is whether the study proposes a metric. Furthermore, there are other topics related to quality as readability, reusability,

Table 7: Topics of primary studies.

\begin{tabular}{|c|c|c|c|c|c|c|c|c|c|c|c|c|c|c|c|}
\hline Reference of primary study & 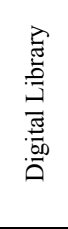 & 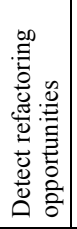 & 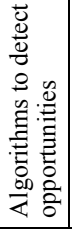 & 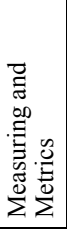 & 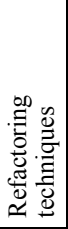 & 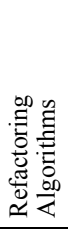 & 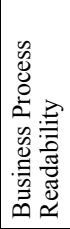 & 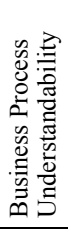 & 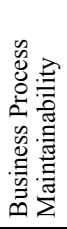 & 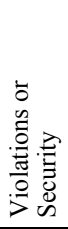 & 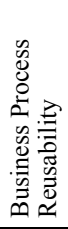 & 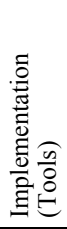 & 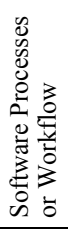 & 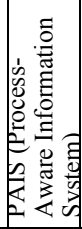 & 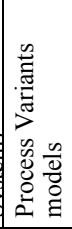 \\
\hline (Weber et al., 2011) & DL1 & 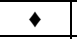 & & & 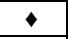 & & 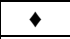 & & 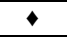 & & & 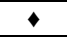 & & 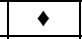 &  \\
\hline (Zeng et al., 2010) & DL1 & 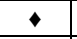 & 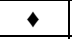 & & $\bullet$ & $\bullet$ & & & & & & & & $\bullet$ & 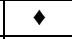 \\
\hline (Hanakawa, 2011) & DL1 & & & & $\bullet$ & & & &  & & & & $\bullet$ & & \\
\hline (Leopold et al., 2010) & DL1 & 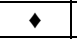 & $\bullet$ & & $\bullet$ & & & $\bullet$ & $\bullet$ & & & & & & \\
\hline (Feineman, 2010) & DL4 & 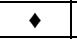 & 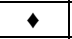 & & & & &  & 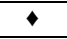 & & & 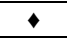 & & & \\
\hline (Yan and Wang, 2009) & DL1 & $\bullet$ & & & & & & & & & & & & & \\
\hline (Vanhatalo et al., 2008) & DL1 & $\bullet$ & & & 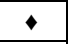 &  & & 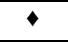 & & & & & & & \\
\hline (Chivers and McDermid, 2006) & DL1 & & & & 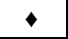 & & & & & $\bullet$ & & & $\bullet$ & & \\
\hline (Wang et al., 2007) & DL1 & 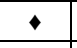 & & & 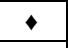 & & & & & & & & & & \\
\hline (Küster et al., 2006) & DL2 & 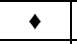 & & & + & & & & & & & & & & \\
\hline (Singh et al., 2007) & DL1 & & & & 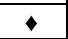 & & & & & & & & 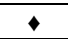 & & \\
\hline
\end{tabular}


security, understandability and maintainability. A topic about whether the study proposes the implementation of its algorithms or not. There is a topic about whether the study is generic as software process or workflow but it can be applied in this context. Also, there is a topic on whether the study is about PAIS (Process-Aware Information System). Finally, there is a topic to indicate whether the study makes refactoring of process variants models.

\section{CONCLUSIONS}

The paper presents a systematic literature review of refactoring techniques and methods to be applied to business process models obtained by reverse engineering. The review has been carried out by following the formal methodology proposed by Kitchenham.

In total, 206 relevant studies were found in four different digital libraries (ACM, SpringerLink, IEEE Xplore and Scopus). 16 of these studies were considered as primary according to the inclusion and exclusion criteria and specific data were collected from them in order to analyze them and to obtain conclusions. After applying a statistical analysis the most valuable findings were the following: (1) as negative aspects: little empirical validation performed. Most of the studies considered only an example of the techniques or methods proposed, and some were proposed as future work to validate their proposals through study cases; (2) as beneficial aspects: growing interest in the field due to increased studies in recent years. It is also an area of research which is not mature enough, so it is interesting to address it.

Particularly, refactoring techniques have not been especially developed to business processes obtained by reverse engineering. Therefore, it may be a possible field in which to make further research efforts since it has not been addressed yet by the research community.

\section{ACKNOWLEDGEMENTS}

This work was supported by the FPU Spanish Program and the R\&D projects ALTAMIRA (PII 2I09-0106-2463), PEGASO/MAGO (TIN200913718-C02-01), MOTERO (JCCM and FEDER, PEII11-0366-9449) and MEDUSAS (CDTI, IDI20090557).

\section{REFERENCES}

Dijkman, R., Gfeller, B., Küster, J. \& Völzer, H. 2011. Identifying refactoring opportunities in process model repositories. Information and Software Technology.

Jeston, J., Nelis, J. \& Davenport, T. 2008. Business Process Management: Practical Guidelines to Successful Implementations, NV, USA, ButterworthHeinemann (Elsevier Ltd.).

Kitchenham, B. \& Charters, S. 2007. Guidelines for performing systematic literature reviews in software engineering. Engineering, 2.

Mens, T., Taentzer, G. \& Müller, D. Year. Challenges in model refactoring. In, 2007.

Mens, T. \& Tourwé, T. 2004. A survey of software refactoring. Software Engineering, IEEE Transactions on, 30, 126-139.

Omg. 2006. Business Process Modeling Notation Specification 1.0 [Online]. Available: http://www. omg.org/bpmn/Documents/OMG_Final_Adopted_BP MN 1-0 Spec 06-02-01.pdf [Accessed].

Opdyke, W. F. 1992. Refactoring: A program restructuring aid in designing object-oriented application frameworks. PhD thesis, University of Illinois at Urbana-Champaign.

Pérez-Castillo, R. \& Fernández-Ropero, M. 2012. Refactoring Business Process Models - A Systematic Review [Online]. Available: http://alarcos.esi.uclm.es/ per/rpdelcastillo/SLR.html [Accessed 21/02/2012 2012].

Pérez-Castillo, R., Fernández-Ropero, M., Guzmán, I. G.R. D. \& Piattini, M. 2011a. MARBLE. A Business Process Archeology Tool. 27th IEEE International Conference on Software Maintenance (ICSM 2011). Williamsburg, VI.

Pérez-Castillo, R., García-Rodríguez De Guzmán, I. \& Piattini, M. 2011b. Business Process Archeology using MARBLE. Information and Software Technology.

Reuters, T. 2011. EndNote ${ }^{\circledR}$. Bibliographies Made Easy TM $\mathrm{http}: / / \mathrm{www}$.endnote.com/.

Weske, M. 2007. Business Process Management: Concepts, Languages, Architectures, Leipzig, Alemania, Springer-Verlag Berlin Heidelberg. 